\title{
Evaluación de la mezcla de sustratos en un cultivo de lechuga (Lactuca sativa L.) var. Verónica
}

\section{Evaluation of substrate mixture in a lettuce yield (Lactuca sativa L.) var. Veronica}

\author{
Andrea J. Reyes-Medina', David Fraile-Robayo², Javier G. Álvarez-Herrera ${ }^{3}$ \\ Recibido para publicación: Marzo 10 de 2018 - Aceptado para publicación: Noviembre 23 de 2018
}

\begin{abstract}
RESUMEN
La producción de hortalizas en sustratos se ha convertido en una gran alternativa. Por lo anterior, se evaluaron diferentes sustratos en un cultivo de lechuga en invernadero. Se utilizó un diseño en bloques completamente al azar (BCA) donde el factor de bloqueo fueron los sustratos y se establecieron tres tratamientos: T1: turba negra $30 \%$, turba de coco $70 \%$, T2: turba negra $70 \%$, turba de coco $30 \%$ y T3: turba negra $50 \%$ y turba de coco $50 \%$, para un total de 9 unidades experimentales, cada unidad experimental estuvo conformada por 10 plantas. Cada 8 días, durante 32 días, se determinó la altura de planta, masa fresca y seca de hojas y raíz, área foliar y cada 2 días, las unidades SPAD y la conductividad estomática (CE). Se presentaron diferencias significativas en las Unidades SPAD en el tiempo con un valor de 20,7. La CE presentó los mayores valores en T3 y T2 con valores de 354,2 y 366,2 mmol $\mathrm{m}^{-2} \mathrm{~s}^{-1}$, respectivamente. El T1 presentó el mayor valor de resistencia estomática (RE) con 7,5 $\mathrm{m}^{2} \mathrm{~s} \mathrm{~mol}^{-1}$. El área foliar y la altura no presentaron diferencias significativas. El T2 a los 15 días después de trasplante mostró los mayores valores de masa fresca de raíz (MFR), masa seca de raíz (MSR) y masa fresca de hojas (MFH) con 3,1 g, 0,106 g y 13,19 g, respectivamente. En la cosecha, el T3 mostró un $10 \%$ y $15 \%$ más de producción de MFH de lechuga, que los tratamientos $\mathrm{T} 2$ y $\mathrm{T} 1$, respectivamente.
\end{abstract}

Palabras clave: Conductancia estomática, hidroponía, turba, turba de coco y resistencia estomática

\begin{abstract}
The production of vegetables on substrates has become a great alternative. Therefore, different substrates were evaluated in a greenhouse lettuce culture. A completely randomized block design (BCA) with three treatments was used as follows: T1: peat 30\% coconut peat $70 \%$, T2: peat $70 \%$ coconut peat $30 \%$ and T3: peat $50 \%$ Coconut peat $50 \%$, for a total of 9 experimental units each consisting of 10 plants. Every 8 days, during 32 days, the height of the plant, fresh and dry mass of leaves and root, leaf area and every 2 days, the SPAD units and the stomatal conductivity (SC) were determined. There were significant differences in the SPAD units in time for day $11 \mathrm{ddt}$ with a value of 20.7. The SC presented the highest values in $\mathrm{T} 3$ and $\mathrm{T} 2$ with values of 354.2 and $366.2 \mathrm{~mol} \mathrm{~m}^{-2} \mathrm{~s}^{-1}$, respectively. T1 presented the highest value of stomatal resistance (RE) with $7.5 \mathrm{~m}^{2} \mathrm{~s} \mathrm{~mol}^{-1}$. The leaf area and height did not show significant differences. T2 at 15 days after transplant showed the highest values of fresh root mass (MFR), dry root mass (MSR) and fresh leaf mass (MFH) with $3.1 \mathrm{~g}$, $0.106 \mathrm{~g}$ and $13.19 \mathrm{~g}$ respectively. In harvest, T3 showed $10 \%$ and $15 \%$ more production of MFH in lettuce than treatments $\mathrm{T} 2$ and $\mathrm{T} 1$, respectively.
\end{abstract}

Key words: Stomatal conductance, hydroponics, peat, coconut peat, and stomatal resistance.

\footnotetext{
${ }^{1}$ Joven investigador. Grupo de Investigaciones Agrícolas (GIA). Facultad de Ciencias Agropecuarias. Universidad Pedagógica y Tecnológica de Colombia (UPTC). Avenida Central del Norte, Tunja, Boyacá. andreu1101@gmail.com

${ }^{2}$ Ingeniero. Agrónomo. Grupo de Investigaciones Agrícolas. Universidad Pedagógica y Tecnológica de Colombia (UPTC). Tunja, Boyacá.

${ }^{3}$ Ph. D. Fisiología Vegetal. Grupo de Investigaciones Agrícolas. Universidad Pedagógica y Tecnológica de Colombia (UPTC). Tunja, Boyacá. Avenida central del norte, 3002141665, jgalvarezh@gmail.com
} 


\section{INTRODUCCIÓN}

Las hortalizas son un producto fundamental para el mercado laboral en el sector agropecuario. El área cosechada a nivel nacional en 2014 superó las 103.000 ha, con una producción cerca de las 1,8 millones de ton y un rendimiento promedio de $17 \mathrm{t} \mathrm{ha}^{-1}$, siendo el segundo cultivo permanente con mejores rendimientos alcanzando una producción total de \$1,4 billones (FINAGRO, 2014).

En Colombia, la agricultura ha experimentado una notable modernización en las zonas de mayor producción y uso intensivo del suelo (Carranza et al., 2009), lo cual ha generado una alta degradación de los suelos, expresada mediante la pérdida de fertilidad, la salinización, la contaminación por agroquímicos, la erosión debida a la eliminación de la cubierta vegetal por el sobrepastoreo o el movimiento constante del suelo (Pérez y Landeros, 2009).

La hidroponía o cultivo sin suelo es una técnica cuya importancia radica en su alta producción por metro cuadrado, gran eficiencia en el uso de agua y la multiplicidad de los cultivos compatibles que brindan a los productores una tecnología diferente de producción (Birgi, 2015). Con esta tecnología se puede favorecer el ambiente en que se desarrolla la raíz de las plantas dado que permite mayor control en cuanto humedad, fertilidad y cambios en las propiedades fisicoquímicas y al mismo tiempo se minimiza los problemas que ocasiona el suelo, tales como la incidencia de plagas y enfermedades (Gutiérrez, 2011).

Los sistemas hidropónicos se han convertido en una alternativa altamente rentable para la producción agrícola destacando su aprovechamiento en la producción de hortalizas (Lacarra y García, 2011). Una de las ventajas que representa el uso de los sustratos regionales es la disponibilidad y menor costo sobre todo los de origen orgánico dada la tendencia al manejo de sistemas de producción con enfoque sustentable (Cruz-Crespo et al., 2012).

Actualmente, la necesidad del uso de materiales para sustratos ha dado auge a las investigaciones a nivel mundial dado que los cultivos hidropónicos con o sin sustrato se les considera como una técnica agronómica amigable con el medio ambiente y con el ser humano, dado que mediante estos sistemas de producción, además de obtener rendimientos altos y productos de calidad, se logra un producto sin presencia de agroquímicos (CruzCrespo et al., 2012). La lechuga es un cultivo que puede adaptarse a climas cuyas temperaturas promedio oscilan entre los 15 y $20{ }^{\circ} \mathrm{C}$, se desarrolla entre los 1.800 y $2.800 \mathrm{msnm}$, con humedades relativas entre $60 \%$ y $70 \%$, y en zonas de baja ocurrencia de vientos (Jaramillo et al, 2016), de ahí que la productividad del cultivo de lechuga así como sus características de color, sabor y textura, dependen en gran medida de la radiación solar, ya que requiere de aproximadamente 12 horas luz por día, al respecto, Fu et al. (2017) mencionan que una alta iluminación provee de mayor energía para la fijación de $\mathrm{CO}_{2}$, lo cual acelera la síntesis de vitamina $\mathrm{C}$ y la asimilación de nitratos en las hojas de las plantas de lechuga.

La producción de lechuga bajo invernadero se ha establecido como un sistema intensivo y continuo, con cierto nivel de tecnificación como riego por goteo, fertirriego, uso de compost y de mulch para control de malezas y el mantenimiento de la humedad (Hernández et al., 2016; Sigurnjak et al., 2016). En este sentido el presente trabajo tiene como objetivo evaluar diferentes sustratos en un cultivo de lechuga (Lactuca sativa L.) bajo condiciones de invernadero.

\section{MATERIALES Y MÉTODOS}

El proyecto se llevó a cabo en el invernadero de Casa de Malla de la Universidad Pedagógica 
y Tecnológica de Colombia sede Tunja (UPTC), ubicada a una altitud de $2.782 \mathrm{msnm}$ con coordenadas $5^{\circ} 32^{\prime} \mathrm{N}$ y $73^{\circ} 23^{\prime} \mathrm{W}$; al interior del invernadero la temperatura promedio durante el estudio fue de $16{ }^{\circ} \mathrm{C}$ y la humedad relativa promedio $(\mathrm{HR})$ del $70 \%$.

Se utilizó un diseño en completamente al azar (BCA) con tres tratamientos correspondientes a los sustratos de la siguiente forma: T1: turba negra 30\% y turba de coco $70 \%$, T2: turba negra $70 \%$ y turba de coco $30 \%$ y T3: turba negra $50 \%$ y turba de coco $50 \%$, con tres repeticiones para un total de 9 unidades experimentales cada unidad conformada por 10 plantas para un total de 90 plantas evaluadas en el ensayo. Los sustratos fueron seleccionados de acuerdo a las características señaladas en la tabla 2. Se utilizó como material lechuga variedad Verónica el cual fue obtenido en un vivero comercial seleccionando aquellas plántulas que presentaron homogeneidad en el desarrollo fisiológico y óptima sanidad. Se trasplantaron a las materas de $2 \mathrm{~L}$ cuando cumplieron 25 días.

Se realizaron las mediciones de Unidades de clorofila Soil Plant Analysis Development (SPAD) con un SPAD-502 Plus (Decagon Devices Inc, Pullman, WA), conductancia estomática y Resistencia estomática mediante un equipo SC-1 Leaf Porometer (Decagon Devices Inc, Pullman, WA), cada tercer día en el invernadero. Adicionalmente, de determinó la altura desde la base del tallo hasta el extremo superior de la hoja con una regla métrica. Las variables masa fresca de hojas (MFH), masa seca de hojas (MSH), masa fresca de raíz (MFR) y masa seca de raíz (MSR) fueron tomadas cada ocho días en el Laboratorio de Fisiología Vegetal de la Universidad Pedagógica y Tecnológica de Colombia sede Tunja con una balanza electrónica Acculab VIC 612 de 0,01g de precisión (Sartorius Spain S.A., Madrid) y área foliar se midió a través del programa ImageJ v. 1.50i.

La fertilización se realizó calculando las dosis en partes por millón (ppm) llevados a gramos de los nutrientes de fertilizantes comerciales y aplicándolo en $100 \mathrm{ml}$ de agua (Tabla 1). Con el fin de establecer mediante modelos el crecimiento de las plantas de lechuga para cada tratamiento, las variables altura, masa fresca y seca de hojas y raíces y el área foliar fueron ajustadas a una curva logística sigmoidea la cual presenta la siguiente ecuación

Tabla 1. Tiempos y dosis de aplicación de fertilizantes en el cultivo de lechuga.

\begin{tabular}{lcc}
\hline Fertilizante & $\begin{array}{c}\text { Dosis (g) por } \\
\text { matera }\end{array}$ & Día de aplicación \\
\hline Ferfos & 3 & Lunes y Miércoles \\
Urea & 1 & Lunes y Miércoles \\
Nitrato de potasio & 19 & Martes y Viernes \\
Nitrato de calcio & 5,5 & Martes y Viernes \\
Sulfato & 6,5 & Jueves \\
Menores & 16 & Sábado \\
\hline
\end{tabular}

En donde $\mathrm{f}(\mathrm{t})$ es la variable medida en función del tiempo, a es el máximo valor de la variable en el tiempo, b es la máxima velocidad de crecimiento que alcanza la variable y c es el día en el cual se alcanza dicha máxima velocidad.

$$
f(t)=\frac{a}{1+e^{-b^{*}(t-c)}}
$$

Para el análisis de datos, se realizó una prueba de normalidad y se eliminaron los datos atípicos o erróneos, luego se llevó a cabo un análisis de varianza (Anova) con cada una de las variables de respuesta, con el fin de establecer las diferencias entre tratamientos. Posteriormente, se efectuó una prueba de Tukey al 5\%. Lo anterior se ejecutó con el paquete estadístico SAS $\mathbb{R}$ V 9.4 (SAS Institute Inc, Cary, NC).

\section{RESULTADOS Y DISCUSIÓN}

El contenido de clorofila en unidades SPAD no presentó diferencias significativas en cada unas de las mediciones en el tiempo para los tratamientos, a excepción de la medición realizada en el día 13 después del trasplante (ddt), en donde el mejor tratamiento fue la mezcla de $50 \%$ de turba negra con un 50\% de turba de coco (Tabla 2), el cual alcanzo un valor de P 0,0139 (10,9 Unidades SPAD) (Tabla 3). Es probable que para esta medición el efecto de los sustratos se haya maximizado, pues es sabido que las turbas poseen una reserva 
limitada de nutrientes (Fernández, 2010), por lo cual es necesario aumentar las cantidades de aplicación durante el proceso de fertilización para generar una homogenización del efecto de los tratamientos. Dado que la medición es hecha en la hoja, los valores de unidades de clorofila son mayores durante el inicio del desarrollo vegetativo, momento en el cual la planta se encuentra en la plenitud del proceso fotosintético. A partir de esta fase hasta el fin del ciclo, las unidades de clorofila se reducen, esta disminución es más acentuada en las menores dosis de $\mathrm{N}$, en función de su menor disponibilidad (Ribeiro da Cunha et al., 2015).

\begin{tabular}{|c|c|c|c|}
\hline \multicolumn{2}{|c|}{$\begin{array}{l}\text { Propiedades } \\
\text { físicas }\end{array}$} & $\begin{array}{l}\text { Turba de } \\
\text { coco }\end{array}$ & $\underset{\text { negra }}{\text { u }} \underset{\text { r b }}{a}$ \\
\hline \multicolumn{2}{|l|}{ Densidad (g/cm3) } & $0,08-0,12$ & $40-80$ \\
\hline \multicolumn{2}{|l|}{ Porosidad Total (\%) } & $80-82$ & $95-98$ \\
\hline \multicolumn{2}{|l|}{ Capacidad de Aireación (\%) } & $20-30$ & $16-58$ \\
\hline \multicolumn{2}{|c|}{ Agua fácilmente disponible (\%) } & $45-60$ & 40- 82 \\
\hline \multicolumn{2}{|c|}{ Granulometría (\% del peso total en $\mathrm{mm}$ ) } & $2,5-5$ & $1-2$ \\
\hline \multicolumn{2}{|c|}{ Conductividad eléctrica en retención $(\mathrm{mS} / \mathrm{cm})$} & $<100$ & $<175$ \\
\hline
\end{tabular}

Se presentaron diferencias significativas entre tratamientos para la conductancia y la resistencia estomática. Para la conductancia estomática (CE) los tratamientos T2 y T3 presentaron diferencia con valores de $\mathrm{P} 0,06$

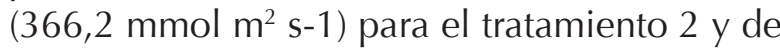
$0,02\left(354,2 \mathrm{mmol} \mathrm{m}^{-2} \mathrm{~s}^{-1}\right)$ para el tratamiento 3 , con respecto al tratamiento 1 . Por su parte, para la RE, el mejor resultado se presentó en el T1, el cual alcanzó un valor de P de 0,05 (7,5 $\left.\mathrm{m}^{2} \mathrm{~s} \mathrm{~mol}^{-1}\right)$. Simultáneamente se presentaron diferencias en el tiempo entre los días 5, 9 y $11 \mathrm{ddt}$ para la conductancia y en los 5, 11 y 12 ddt para la RE (Tabla 3 ) días que coinciden con los resultados de diferencias entre tratamientos. Este comportamiento pudo presentarse debido a que pese a las características de retención de humedad de los sustratos y las mezclas de los mismos las altas temperaturas en el aire y las hojas pueden dar como resultado un aumento en la CE dado que las plantas fueron expuestas totalmente al sol. Bajo condiciones de adecuada disponibilidad de agua, la intensidad lumínica determina en gran medida la apertura estomática. Existen moléculas fotorreceptoras en las células oclusivas de los estomas, del grupo de las fototropinas, que detectan la señal de luz, lo cual incrementa la apertura estomática (Garcés, 2013). En cultivos como el sorgo existen reportes de reducción en los valores de CE cuando las plantas son sometidas a estrés por baja luminosidad (Hattori et al., 2007).

Tabla 3. Valores de probabilidad (P) de ocurrencia de igualdad estadística entre los tratamientos arrojados en el Anova para las variables Unidades SPAD, conductancia estomática y resistencia estomática evaluadas en plantas de lechuga cultivadas en diferentes sustratos

\begin{tabular}{clcc}
\hline $\begin{array}{c}\text { Tiempo } \\
(\mathbf{d d t})\end{array}$ & SPAD & $\mathbf{C E}\left(\mathbf{m m o l} \mathbf{~ m}^{-\mathbf{2}} \mathbf{s}^{-\mathbf{1}}\right)$ & $\mathbf{R E}\left(\mathbf{m}^{\mathbf{2}} \mathbf{s} \mathbf{~ m o l}^{-\mathbf{1}}\right)$ \\
\hline 1 & $0,07^{*}$ & 0,70 & 0,33 \\
3 & 0,16 & 0,67 & 0,71 \\
5 & $0,03^{*}$ & $0,06^{*}$ & $0,05^{*}$ \\
7 & 0,13 & 0,18 & 0,46 \\
9 & 0,27 & 0,00 & $0,02^{*}$ \\
11 & 0,86 & 0,78 & 0,80 \\
13 & $0,01^{*}$ & 0,16 & 0,17 \\
15 & 0,21 & 0,62 & 0,56 \\
17 & 0,36 & $0,02^{*}$ & 0,08 \\
19 & $0,10^{*}$ & 0,36 & 0,42 \\
21 & 0,61 & $0,00^{*}$ & $0,00^{*}$ \\
23 & 0,14 & 0,57 & $0,00^{*}$ \\
25 & $0,00^{*}$ & 0,22 & 0,16 \\
27 & 0,43 & 0,51 & 0,56 \\
29 & 0,22 & 0,99 & 0,99 \\
\hline
\end{tabular}

ddt: días después del trasplante; CE: Conductancia estomática; RE: Resistencias estomática;* indican diferencias significativas con $\mathrm{P} \leq 0,05$.

Al analizar los valores del SPAD en el tiempo para las plantas de lechuga, estos presentaron diferencias significativas entre los días 1, 5, 13, 19 y 25 (Tabla 4), lo cual ocurre por los cambios fenológicos y el crecimiento de las lechugas, ya que para el día 11 ddt se presentó la mayor cantidad de unidades SPAD con un valor de P 0,8627 (20,7 Unidades SPAD), por lo que es probable que las concentraciones de nitrógeno en la hoja hayan aumentado junto con la densidad de clorofila en los cloroplastos, dado que la mayoría del nitrógeno foliar se encuentra contenido en las moléculas de clorofila, de tal forma que la concentración de clorofila en las hojas sea un índice de su contenido de nitrógeno. A partir de los 11 ddt, las unidades SPAD en las plantas de lechuga disminuyeron, lo cual se atribuye a que desde este momento comienza una fase de Ilenado la cual evita la acumulación de clorofilas en los tejidos y da paso al proceso de elongación celular. 
Por el contrario, evaluaciones realizadas en coliflor con diferentes fuentes nitrogenadas no presentaron diferencias significativas en cuanto a los niveles de medición de clorofila (Rivacoba, 2013).

Tabla 4. Valores de probabilidad (P) de ocurrencia de igualdad estadística entre los tratamientos arrojados en el Anova para las variables destructivas evaluadas en plantas delechuga cultivadas en diferentes sustratos

\begin{tabular}{ccccccc}
\hline $\begin{array}{c}\text { Tiempo } \\
\text { (ddt) }\end{array}$ & Altura & MFH & MSH & MFR & MFSR Á r e a \\
foliar
\end{tabular}

MFH: Masa fresca de hojas; MSH: Masa seca de hojas; MFR: masa fresca de raíz; MSR: Masa seca de raíz; AF: área foliar; Altura; ${ }^{*}$ indican diferencias significativas con $\mathrm{P} \leq 0,05$.

Se presentaron diferencias significativas entre tratamientos para la conductancia y la resistencia estomática. Para la conductancia estomática (CE) los tratamientos T2 y T3 presentaron diferencia con valores de $\mathrm{P} 0,06$ $\left(366,2 \mathrm{mmol} \mathrm{m}^{2} \mathrm{~s}^{-1}\right)$ para el tratamiento 2 y de $0,02\left(354,2 \mathrm{mmol} \mathrm{m}^{2} \mathrm{~s}^{-1}\right)$ para el tratamiento 3 , con respecto al tratamiento 1 . Por su parte, para la $\mathrm{RE}$, el mejor resultado se presentó en el T1, el cual alcanzó un valor de P de 0,05 (7,5 $\left.\mathrm{m}^{2} \mathrm{~s}^{-1} \mathrm{~mol}^{-1}\right)$. Simultáneamente se presentaron diferencias en el tiempo entre los días 5, 9 y 11 ddt para la conductancia y en los 5, 11 y 12 ddt para la RE (Tabla 3) días que coinciden con los resultados de diferencias entre tratamientos. Este comportamiento pudo presentarse debido a que pese a las características de retención de humedad de los sustratos y las mezclas de los mismos las altas temperaturas en el aire y las hojas pueden dar como resultado un aumento en la CE dado que las plantas fueron expuestas totalmente al sol. Bajo condiciones de adecuada disponibilidad de agua, la intensidad lumínica determina en gran medida la apertura estomática. Existen moléculas fotorreceptoras en las células oclusivas de los estomas, del grupo de las fototropinas, que detectan la señal de luz, lo cual incrementa la apertura estomática (Garcés, 2013).
En cultivos como el sorgo existen reportes de reducción en los valores de CE cuando las plantas son sometidas a estrés por baja luminosidad (Hattori et al., 2007). Las variables de masa fresca de raíz (MFR), masa seca de raíz (MSR) y masa fresca de hojas (MFH) presentaron diferencias significativas entre tratamientos (Tabla 4). Para las tres variables el T2 presentó mayor significancia con un valor de $\mathrm{P}$ de $0,074(3,1 \mathrm{~g})$ para MFR, 0,104 valor de $\mathrm{P}(0,106 \mathrm{~g})$ en MSR y 0,013 valor de $\mathrm{P}(13,19 \mathrm{~g})$ para MFH. Lo anterior se debe posiblemente a la facultad de la turba negra de favorecer el enraizamiento y producción de fitomasa (Álvarez-Herrera et al. 2007).

Además, los resultados concuerdan con lo encontrado por Guerrero et al. (2014) quienes hallaron diferencias significativas en los tratamientos de turba de coco mezclado con cascarilla de arroz en diferentes proporciones, así como también concuerdan con Astiz et al. (2010) quienes mencionan que al variar las proporciones entre los sustratos varían también sus propiedades, tales como la capacidad de aireación y retención de agua y por ende el desarrollo de la planta. En cuanto al crecimiento, el modelo de crecimiento logístico sigmoideo fue el que presentó mayor ajuste similar a lo demostrado por Carranza et al. (2009). En el se aprecian al comienzo una intensa división y expansión de células meristemáticas, y finaliza con el ralentizamiento del crecimiento en los últimos días de vida del cultivo. Al respecto, se apreciaron notables variaciones en los valores de a, b, c (Tabla 5) como resultado de la aplicación de los tratamientos. Del mismo modo, Campos (2009) considera que los sustratos que se encuentran en un rango de $10 \%$ a $30 \%$ de capacidad de aireación como los seleccionados para este ensayo son óptimos para el cultivo de hortalizas. Según Soto (2007), la aireación es indispensable debido a que el sistema radicular de la planta se encuentra en un volumen limitado para su desarrollo y al ser 
un sistema intensivo de producción presenta una alta actividad metabólica y de crecimiento, por lo cual el requerimiento de oxígeno es más alto. Sumado a lo anterior, el valor de temperatura de acuerdo con Resh (2006), no debe exceder los $30{ }^{\circ} \mathrm{C}$ para evitar que se afecte el crecimiento de las plantas. La temperatura mínima registrada durante el día a lo largo del presente ensayo fue de $12{ }^{\circ} \mathrm{C}$, que de acuerdo con el mismo autor, es favorable también para el desarrollo de la planta de lechuga, lo cual hace que esta obtenga una adecuada ganancia de masa y por consiguiente una buena calidad.

No se presentaron diferencias significativas entre tratamientos para el área foliar y la altura (Tabla 4). De acuerdo con Araméndiz-Tatis et al. (2013), el aporte nutricional de los sustratos mezclados con la turba aportan nitrógeno y fósforo durante las primeras semanas del cultivo lo que favorece un buen desarrollo de las plántulas. Resultados similares fueron obtenidos por Arenas et al. (2002) al reportar en tomate, plántulas de menor tamaño con sustratos compuestos con un $50 \%$ de fibra de coco. No obstante, los valores a, b, y c (Tabla 5) extraídos de los modelos de crecimiento del área foliar y de la altura muestran el leve incremento en las primeras etapas de crecimiento ocasionado probablemente por las ventajas de la aireación y el aporte nutricional que realizan los sustratos en las primeras semanas de trasplante (Pinto et al. 2014), lo cual favorece los procesos de división celular activa, posteriormente, ocurre un aumento en la elongación celular, lo que genera que el crecimiento aumente de forma significativa para finalmente llevar a la fase de maduración en donde el crecimiento se ralentiza (Ardila 2010).

Los sustratos actúan como reserva de nutrientes (Guerrero et al., 2014); así, el crecimiento de las plantas en altura y el aumento de área foliar, es un indicador de que el medio proporcionó las cantidades necesarias y adecuadas de elementos minerales, lo que permitió el crecimiento vigoroso de las lechugas, al respecto, pudo suceder que el contenido nutricional presente en los sustratos estuvo tan homogeneizado que no generó

Tabla 5. Parámetros del modelo de crecimiento logístico sigmoideo encontrados para los diferentes sustratos empleados en lechuga

\begin{tabular}{|c|c|c|c|c|c|c|c|}
\hline Parámetro & Tratamiento & Altura & MFH & MSH & MFR & MFSR & Área foliar \\
\hline & & $\begin{array}{c}\text { Máximo } \\
\text { valor }(\mathbf{c m})\end{array}$ & & Máxim & valor (gr) & & $\begin{array}{c}\text { Máximo } \\
\text { valor }\left(\mathbf{c m}^{2}\right)\end{array}$ \\
\hline \multirow[t]{2}{*}{ a } & $\begin{array}{l}\text { T1 } \\
\text { T2 } \\
\text { T3 }\end{array}$ & $\begin{array}{l}18,86 \\
12,25 \\
17,38\end{array}$ & $\begin{array}{l}576,3 \\
296,6 \\
178,8\end{array}$ & $\begin{array}{c}13,62 \\
11,23 \\
6,94\end{array}$ & $\begin{array}{c}6231974 \\
38,27 \\
32,78\end{array}$ & $\begin{array}{l}1,96 \\
1,82 \\
2,42\end{array}$ & $\begin{array}{c}3108,5 \\
1954,7 \\
1771\end{array}$ \\
\hline & & $\begin{array}{c}\text { M á x i m a } \\
\text { tasa de cre- } \\
\text { c i m i e n to } \\
\left(\mathrm{cm} \mathrm{día}{ }^{-1}\right)\end{array}$ & \multicolumn{4}{|c|}{ Máxima tasa de crecimiento (g día-1) } & $\begin{array}{c}\text { M á x i m a } \\
\text { tasa de cre- } \\
\text { c i m i e n to } \\
\left(\mathrm{cm}^{2} \text { día }^{-1}\right) \\
\end{array}$ \\
\hline $\mathrm{b}$ & $\begin{array}{l}\text { T1 } \\
\text { T2 } \\
\text { T3 }\end{array}$ & $\begin{array}{l}0,103 \\
0,163 \\
0,116\end{array}$ & $\begin{array}{l}0,16 \\
0,17 \\
0,26\end{array}$ & $\begin{array}{l}0,18 \\
0,20 \\
0,27\end{array}$ & $\begin{array}{l}0,16 \\
0,29 \\
0,37\end{array}$ & $\begin{array}{l}0,23 \\
0,38 \\
0,41\end{array}$ & $\begin{array}{l}0,23 \\
0,27 \\
0,28\end{array}$ \\
\hline \multicolumn{8}{|c|}{ Tiempo (días) } \\
\hline $\mathrm{b}$ & $\begin{array}{l}\text { T1 } \\
\text { T2 } \\
\text { T3 }\end{array}$ & $\begin{array}{l}18,63 \\
12,26 \\
16,02\end{array}$ & $\begin{array}{l}39,08 \\
32,58 \\
26,05\end{array}$ & $\begin{array}{l}30,78 \\
28,13 \\
23,62\end{array}$ & $\begin{array}{l}107,4 \\
23,13 \\
22,32\end{array}$ & $\begin{array}{l}27,25 \\
22,23 \\
22,65\end{array}$ & $\begin{array}{l}27,62 \\
24,26 \\
23,75\end{array}$ \\
\hline
\end{tabular}

T1: turba negra $30 \%$ y turba de coco $70 \%$, T2: turba negra $70 \%$ y turba de coco $30 \%$ y T3: turba negra $50 \%$ y turba de coco $50 \%$. a es el máximo valor de la variable en el tiempo, b es la máxima velocidad de crecimiento que alcanza la variable y c es el día en el cual se alcanza la máxima velocidad de crecimiento 
diferencias entre tratamientos con respecto al crecimiento y desarrollo de las plantas.

\section{CONCLUSIONES}

A partir de los $11 \mathrm{ddt}$, el contenido de clorofila en unidades SPAD en las plantas de lechuga disminuyó, lo que se atribuyó a la finalización del ciclo vegetativo y por ende la reducción de la disponibilidad de nitrógeno. El área foliar y la altura no presentaron diferencias significativas para los sustratos evaluados.

La mezcla de turba negra $70 \%$ y turba de coco $30 \%$ presentaron los mayores valores de masa fresca y seca de raíces así como mayor masa fresca de hojas, incrementando la fitomasa, dado que la combinación de diferentes proporciones las proporciones entre los sustratos varían también sus propiedades como la capacidad de aireación y retención de agua teniendo un efecto directo en el desarrollo de la planta, por lo que se puede concluir que el T3 fue el mejor tratamiento para el crecimiento de lechuga.

\section{REFERENCIAS}

Álvarez-Herrera, J., Rodríguez, S. y Chacón, E. 2007. Efecto de diferentes tamaños de esqueje y sustratos en la propagación del romero (Rosmarinus officinalis L.). Agronomía Colombiana. 25(2): 224-23

Araméndiz-Tatis, H., Cardona-Ayala, C. y Correa-Álvarez, E. 2013. Efecto de diferentes sustratos en la calidad de plántulas de berenjena (Solanum melongena L.). Revista Colombiana de Ciencias Hortícolas. 7(1): 55-61

Ardila, H. 2010. Estudio del crecimiento de la planta y del fruto de tres híbridos de tomate (Solanum lycopersicum L.) en tiempo fisiológico. Tesis de especialización. Facultad de Agronomía. Universidad Nacional deColombia. Bogotá, Colombia.
Arenas, M., Vavrina, C.S., Cornell, J.A., Hanton, E.A. y Hochmuth, G.J. 2002. Coir as an alternative to peat in media for tomato transplant production. HortScience 37(2): 309-312.

Astiz, M., Uribarri, J., Aguado, A., Apesteguía, M. y Sádaba, S. 2010. Tomate hidropónico, Ed. Navarra Agropecuaria. Lima, Perú. 42 p.

Birgi, A. 2015. Producción hidropónica de hortalizas de hoja. Instituto nacional de tecnología agropecuaria. Estación experimental Agropecuaria Santa Cruz. Área de producción Agrícola. Santa Cruz, Argentina.

Campos, M. 2009. Efecto de la inoculación de sustratos con Trichoderma spp. sobre el crecimiento y producción de plantas de chile dulce (Capsicum annuum Linn), bajo ambiente protegido. Tesis. Instituto Tecnológico de Costa Rica. Costa Rica.

Carranza, C., Lanchero, O., Miranda, D. y Chaves, B. 2009. Análisis del crecimiento de lechuga (Lactuca sativa L.) 'Batavia' cultivada en un suelo salino de la Sabana de Bogotá. Agronomía Colombiana. 27: 41-48.

Cruz-Crespo, E., Can-Chulim, A., SandovalVilla, M., Bugarín-Montoya, R., Robles-Bermúdez, A., y Juárez-López, P. 2012. Sustratos en la horticultura. Revista Bio Ciencias. 2(2): 17-26.

Fernández, M. 2010. Evaluación de sustratos de fibra de madera de pino frente a sustratos convencionales en cultivo hidropónico de tomate. Tesis. Universidad Pública de Navarra. Pamplona. España.

FINAGRO, 2014. Perspectiva del sector agropecuario Colombiano. Equipo TécnicodePresidencia.Bogotá, Colombia.

Fu, Y., Li, H., Yu, J., Liu, H., Cao, Z., Manukovsky, $N$. and Liu, H. 2017. Interaction effects of light intensity and nitrogen concentration on growth, photosynthetic characteristics and quality of lettuce (Lactuca sativa L. Var. youmaicai). Scientia Horticulturae 214: 51-57 
Garcés, G. 2013. Influencia de las altas temperaturas, la baja luminosidad y la época de siembra sobre el comportamiento fisiológico de dos cultivares de arroz (Oryza sativa L.). Tesis de Maestría. Universidad Nacional de Colombia Facultad de Agronomía, Escuela de posgrados Bogotá, Colombia.

Guerrero, E., Revelo, J., Benavides, O., Chaves, G y Moncayo, C. 2014. Evaluación de sustratos en un cultivo de lechuga bajo un sistema hidropónico en el municipio de Pasto. Revista de Ciencias Agrícolas 31(1): 3-16.

Gutiérrez, J. 2011. Producción hidropónica de lechuga con y sin recirculación de solución nutritiva. Tesis maestro en ciencias en horticultura. Universidad Autónoma de México. 66 p.

Hattori, T., Sonobe, K., Inanaga, S., An, P., Tsuji, W., Araki, H., Eneji, A. and Morita, S. 2007. Short term stomatal responses to light intensity changes and osmotic stress in sorghum seedlings raised with and without silicon. Environmental and Experimental Botany 60, 177-182.

Hernández, T., Chocano, C., Moreno, J. y García, C. 2016. Use of compost as an alternative to conventional inorganic fertilizers in intensive lettuce (Lactuca sativa L.) crops-Effects on soil and plant. Soil and Tillage Research 160: 14-22.

Jaramillo, J., Aguilar, P., Aguilar, P., Julián., Tamayo, E., Arguello., O., Guzmán, M y CORPOICA. 2016. Modelo Tecnológico para el cultivo de Lechuga bajo Buenas Prácticas Agrícolas en el Oriente Antioqueño. Medellín, p 40.
Perez, A y Landeros, C. 2009. Agricultura y deterioro ambiental. Elementos 73: 19-25.

Pinto, E., Almeida A., Aguiar, A. and Ferreira, E. 2014. Changes in macrominerals, trace elements and pigments content during lettuce (Lactuca sativa L.) growth: Influence of soil composition. Food Chemistry 152: 603-611.

Resh, H. 2006. Cultivos hidropónicos. Madrid. Ed 5ta. Mundo Prensa. 364 p.

Rivacoba, L. 2013. Evaluación de medidas de nitrógeno en la planta para su uso como sistema de recomendación de abonado nitrogenado en el cultivo de coliflor. Tesis Doctoral. Facultad de Ciencias. Estudios Agroalimentarios e Informática. Universidad de La Rioja. España.

Ribeiro da Cunha, A., Leoschua, K., De Pádua Sousa, A y Martinez, R. 2015. Indice SPAD en el crecimiento y desarrollo de plantas de lisianthus en función de diferentes dosis de nitrógeno en ambiente protegido. IDESIA. 33(2): 97-105.

Sigurnjak, I., Michels, E., Crappé, S., Buysens, S., Tack, F. and Meers, E. 2016. Utilization of derivatives from nutrient recovery processes as alternatives for fossilbased mineral fertilizers in commercial greenhouse production of Lactuca sativa L. Scientia Horticulturae 198: 267-276.

Soto, F. 2007. Boletín del programa nacional sectorial de producción agrícola bajo ambientes protegidos. PRONAP. Costa Rica. 8p. 